\title{
Photoisomerization of 2-(2'-Hydroxyphenyl)benzoxazole. Formation and Decay of the Trans-Keto Tautomer in Dry and in Water-Containing 3-Methylpentane
}

\author{
Jens S. Stephan and Karl H. Grellmann* \\ Abteilung Spektroskopie und Photochemische Kinetik, POB 2841, Max-Planck-Institut fur Biophysikalische \\ Chemie, D-37018 Göttingen, Germany
}

Received: February 3, $1995^{8}$

\begin{abstract}
2-(2'-Hydroxyphenyl)benzoxazole, like other $o$-hydroxyphenylbenzazoles, forms after excited-state intramolecular proton transfer the trans-keto tautomer, ${ }^{1} K_{\mathrm{tr}}$. In dry, inert solvents this tautomer is relatively longlived and decays in a bimolecular reaction by double proton transfer. Traces of water shorten the lifetime of ${ }^{1} K_{\mathrm{tr}}$ drastically due to proton-catalyzed re-enolization. In former investigations, the formation of ${ }^{1} K_{\mathrm{tr}}$ has been overlooked by us. The quantum yield of trans-keto formation is temperature dependent and becomes negligibly small below $\sim 160 \mathrm{~K}$.
\end{abstract}

\section{Introduction}

The excited-state intramolecular proton-transfer (ESIPT) reaction of $o$-hydroxyphenylbenzazoles $(1)$ has been studied

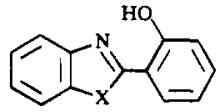

cis - enol

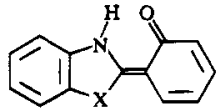

cis - keto

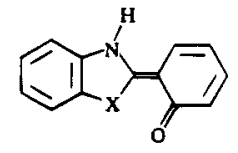

trans - keto

\section{SCHEME 1}

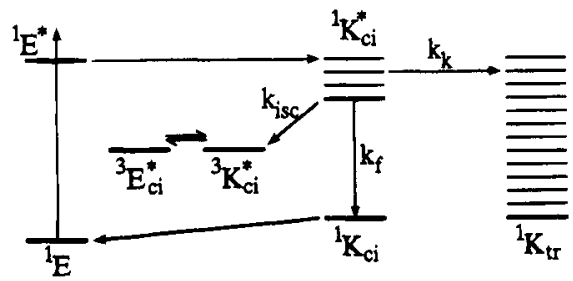

extensively in recent years. ${ }^{1}$ In inert solvents, the cis-enol, ${ }^{1} \mathrm{E}$, (cf. Scheme 1) is the most stable form in the ground state. After excitation to the first excited singlet state, ${ }^{1} \mathrm{E}^{*}$, ESIPT takes place in the subpicosecond time range, yielding the excited cisketo form, ${ }^{1} \mathrm{~K}^{*}$ ci, which emits a strongly Stokes-shifted fluorescence. In the case of 2-( $2^{\prime}$-hydroxyphenyl)benzoxazole $(\mathbf{1}$, $\mathrm{X}=\mathrm{O} ; \mathrm{HBO}$ ), Itoh and Fujiwara ${ }^{2}$ observed in flash experiments a transient absorption to which a double-exponential decay was fitted with the lifetimes $\tau_{\mathrm{a}}=0.26 \mu \mathrm{s}$ and $\tau_{\mathrm{b}}=10.9 \mu \mathrm{s}$ at room temperature. The long-lived transient with $\tau_{\mathrm{b}}=10.9 \mu$ s could be quenched by oxygen and was therefore assigned to the triplet state of HBO which is formed after ESIPT from ${ }^{\prime} \mathrm{K}^{*}$. . The short-lived transient $\left(\tau_{\mathrm{a}}=0.26 \mu \mathrm{s}\right)$ Itoh and Fujiwara ${ }^{2}$ assigned to the cis-keto form in its ground state, ${ }^{1} \mathrm{~K}_{\mathrm{c}}$.

In our laboratory we found in nonpolar HBO solutions a transient absorption ${ }^{3.4}$ whose nonexponential decay kinetics depended strongly on the transient concentration. We did not observe an oxygen-insensitive transient and proposed that the nonexponential decay was due to second-order triplet-triplet annihilation (TTA). The detection of a delayed fluorescence at high transient concentrations ${ }^{5}$ corroborated this assumption. The observation of a dual phosphorescence ${ }^{5}$ revealed that after ESIPT the triplet states of the keto form, ${ }^{3} \mathrm{~K}^{*}$ ii , and of the enol form, ${ }^{3} \mathrm{E}^{*}$ ci, are populated via ${ }^{1} \mathrm{~K}{ }_{\mathrm{ci}}$.

In 1990 Brewer et al. ${ }^{6}$ observed the formation of the transketo isomer, ${ }^{1} \mathrm{~K}_{\mathrm{tr}}$ (cf. Scheme 1$)$ in the case of 2 -( $2{ }^{\prime}$-hydroxyphenyl)benzthiazole $(1, X=S ; H B T)$. This oxygen-insensitive transient decays in a second-order reaction ${ }^{7,8}$ back to the stable enol form by intermolecular double proton transfer. ${ }^{7}$ With 2-( $2^{\prime}$ hydroxyphenyl)-3-H-indole $\left(1, \mathrm{X}=\mathrm{C}\left(\mathrm{CH}_{3}\right)_{2} ; \mathrm{HBC}\right)$ too, the corresponding trans-keto form has been observed recently. ${ }^{9}$ It

\footnotetext{
${ }^{\otimes}$ Abstract published in Advance ACS Abstracts, June 1, 1995
}

also decays in dry nonpolar solvents by double proton transfer, as shown schematically in Figure 1A. In both compounds, the absorption spectra of the trans-keto tautomers and of the triplet states strongly overlap. Furthermore, it turned out, that the reenolization of the trans-keto form can be very efficiently catalyzed by protic additives such as methanol or water, ${ }^{9}$ whereby the trans-keto lifetime is shortened several orders of magnitude and the decay becomes first order. If methanol is the proton donor, two alcohol molecules are involved in the re-enolization (cf. Figure 1B). The formation of ${ }^{1} \mathrm{~K}_{\mathrm{tr}}$ is thermally activated: At ambient temperatures the quantum yield of ${ }^{1} \mathrm{~K}_{\mathrm{tr}}$ formation is high, below $\sim 140 \mathrm{~K}$ it becomes negligible. ${ }^{7.9}$

These recent observations, especially the proton-catalyzed trans-keto re-enolization, led us to reexamine $\mathrm{HBO}$ in nonpolar solution and, indeed, it turned out that $\mathrm{HBO}$ is no exception in that it forms, like HBT and HBC, the trans-keto tautomer. Its formation has been overlooked ${ }^{3.4}$ because in the inert solvent 3-methylpentane (3MP) it is difficult to distinguish the transketo decay from the triplet-state decay and because traces of protic impurities drastically shorten the trans-keto lifetime, as we will show in this letter.

\section{Experimental Section}

Solutions of HBO in 3MP were excited with an excimer laser $\left(308 \mathrm{~nm}, \sim 500 \mathrm{~mJ} / \mathrm{cm}^{2}\right)$. The purification of $\mathrm{HBO}$ and $3 \mathrm{MP}$, the registration and analysis of the transient absorption have been described. ${ }^{4}$ To add small amounts of water to a degassed and dried solution, $6.0 \mathrm{~mL}$ of saturated water vapor $\left(2^{\circ} \mathrm{C}\right)$ were condensed into $4.0 \mathrm{~mL}$ of an $\mathrm{HBO}$ solution, as described in detail in ref 9.

\section{Results and Discussion}

In Figure 2A the decay of a transient absorption, $A_{\mathrm{obs}}$, is shown which one observes after laser-flash excitation $(308 \mathrm{~nm})$ 


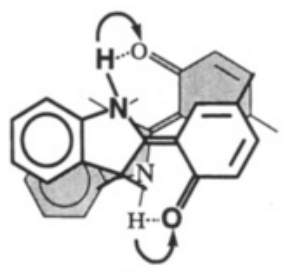

A

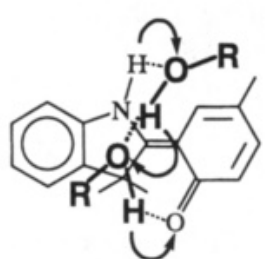

B
Figure 1. (A) Re-enolization of the trans-keto tautomer of HBC by mutual hydrogen exchange (double proton transfer). (B) Re-enolization by proton catalysis (triple proton transfer).
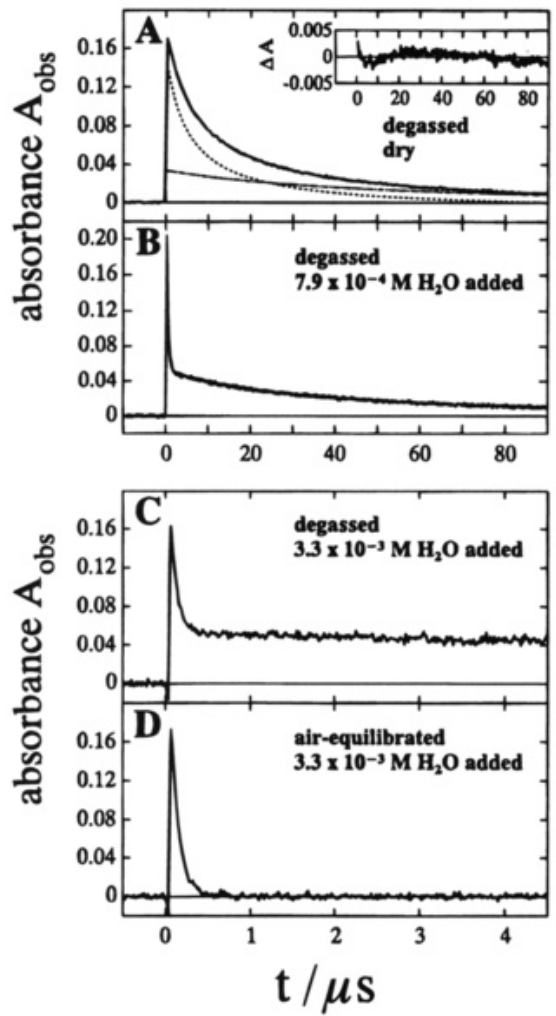

Figure 2. Transient absorption observed after excitation of a $1 \times 10^{-4}$ $\mathrm{M}$ solution of $\mathrm{HBO}$ in $3 \mathrm{MP}$ at $280 \mathrm{~K}$. Excitation wavelength $308 \mathrm{~nm}$, monitoring wavelength $430 \mathrm{~nm}$, optical path length $1 \mathrm{~cm}$. Eight decay traces were sampled and averaged. (A) Dry 3MP. Insert: residuals of a fit of a mixed first and second order (cf. eq 1) plus a second-order (eq 2) decay law with the fixed parameters $k_{1}=7.5 \times 10^{3} \mathrm{~s}^{-1}$ and $\theta_{2}{ }^{t}$ $=4.8 \times 10^{5} \mathrm{~s}^{-1}$ and the free parameters $A^{\circ}{ }_{t}=0.034, A^{\circ}{ }_{k}=0.16$ and $\theta_{2}{ }^{k}=1.1 \times 10^{6} \mathrm{~s}^{-1}$. The fixed parameters $k_{1}$ and $\theta_{2}{ }^{t}$ were taken from a mixed first- and second-order fit to the curve in Figure 2B, neglecting the decay between $t=0$ and $t=3 \mu \mathrm{s}$. The dotted line shows the calculated ${ }^{1} \mathrm{~K}_{\mathrm{tr}}$ contribution to $A_{\mathrm{obs}}$, the dash-dotted line the ${ }^{3} \mathrm{HBO}^{*}$ contribution. (B) Same solution as A, with $7.9 \times 10^{-4} \mathrm{M} \mathrm{H}_{2} \mathrm{O}$ added. (C) Same solution as B with additional water (total $3.3 \times 10^{-3} \mathrm{M}$ ). (D) Same solution as $\mathrm{C}$, but air equilibrated.

of a $1 \times 10^{-4} \mathrm{M}$ solution of $\mathrm{HBO}$ in dried and degassed 3MP. This absorbance decay is composed of two components, one of which is proton-sensitive, the other one is oxygen sensitive: Addition of $8 \times 10^{-4} \mathrm{M} \mathrm{H}_{2} \mathrm{O}$ (Figure 2B) drastically shortens the lifetime of about three quarters of the total absorbance, the rest remains apparently unaffected. Further addition of water (Figure 2C) shortens the lifetime of the proton-sensitive transient only slightly more. If the water-containing solution (Figure 2C) is air equilibrated, the long-lived transient is quenched, the lifetime of the short-lived transient is not affected (Figure 2D).

On the basis of the above-mentioned observations with HBT and $\mathrm{HBC},{ }^{7,9}$ we assign the proton-quenched transient to the trans-keto tautomer ${ }^{1} \mathrm{~K}_{\mathrm{tr}}$ (absorbance $A_{\mathrm{k}}$ ) and the oxygenquenched transient to the $\mathrm{HBO}$ triplet states ${ }^{3} \mathrm{E}^{*}$ ci and ${ }^{3} \mathrm{~K}^{*}{ }_{\mathrm{ci}}$

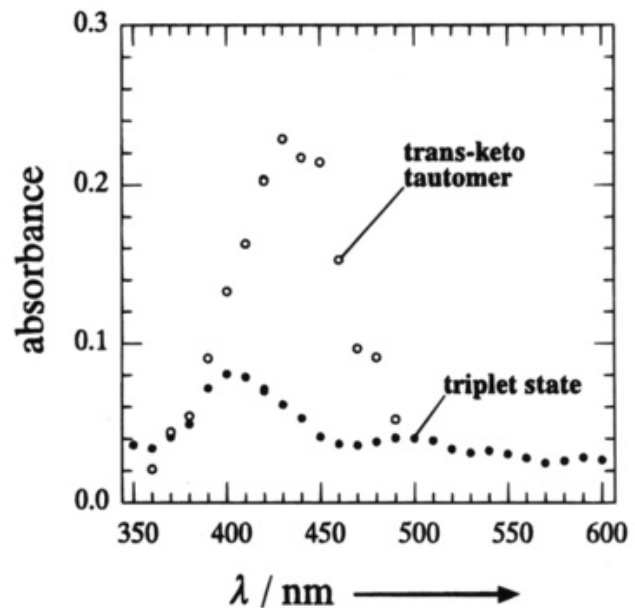

Figure 3. Transient absorption spectrum of the HBO trans-keto tautomer ${ }^{1} \mathrm{~K}_{\mathrm{tr}}(\mathrm{O})$ and of the triplet state ${ }^{3} \mathrm{HBO}^{*}(\bullet)$ at $280 \mathrm{~K}$. The spectra were determined with a degassed, $3.3 \times 10^{-3} \mathrm{M} \mathrm{H}_{2} \mathrm{O}$ containing solution of $\mathrm{HBO}$ in 3MP, from traces like the one in Figure $2 \mathrm{C}$, monitored at different wavelengths in $10 \mathrm{~nm}$ steps (bandwidth $2 \mathrm{~nm}$ ). The triplet absorbance at time zero, $A^{\circ}$, was determined simply by linear extrapolation of the slow-decay trace. The trans-keto absorbance was assumed to be the difference between the total absorbance at zero time and the triplet absorbance: $A^{\circ}{ }_{k}=A^{\circ}{ }_{\text {obs }}-A^{\circ}{ }_{t}$

(absorbance $A_{t}$ ). It has been shown $n^{5,10,11}$ that the two HBO triplet states are, by chance, isoenergetic and in equilibrium with each other. This equilibrium is established and maintained by proton tunneling. ${ }^{10,11}$ The much slower decay of the two equilibrium-coupled triplet states (together designated here $\left.{ }^{3} \mathrm{HBO}^{*}\right)$ comes about by phosphorescence $\left(k_{\mathrm{P}}\right)$, radiationless deactivation $\left(k_{\mathrm{nr}}\right)$ and TTA $\left(k_{\mathrm{tta}}\right)$. Hence, the absorbance decay of ${ }^{3} \mathrm{HBO} *$ is mixed first- and second-order:

$$
\mathrm{d} A_{r} / \mathrm{d} t=-\left(k_{1} A_{t}+\theta_{2}{ }^{t} A_{t}{ }^{2}\right)
$$

where $k_{1}=k_{\mathrm{P}}+k_{\mathrm{nr}}, \theta_{2}{ }^{t}=k_{\mathrm{ta}} / \epsilon_{t} d$ and $A_{t}=\left[{ }^{3} \mathrm{HBO}^{*}\right] \epsilon_{t} d . \epsilon_{t}$ is the extinction coefficient of ${ }^{3} \mathrm{HBO}^{*}$ at the monitoring wavelength, $d$ is the optical path length. If the initial absorbance, $A^{\circ}{ }_{\text {obs }}$, is high, like in Figure 2, the TTA contribution to the decay is substantial during the first $30-40 \mu \mathrm{s}$.

The trans-keto tautomer decays in dry 3MP most probably in a second-order reaction (rate constant $k_{2}$ ) as in the case of $\mathrm{HBT}^{7}$ and HBC: ${ }^{9}$

$$
\mathrm{d} A_{\mathrm{k}} / \mathrm{d} t=-\theta_{2}{ }^{k} A_{k}{ }^{2}
$$

where $\theta_{2}{ }^{k}=k_{2} / \epsilon_{k} d, A_{k}=\left[{ }^{1} K_{\mathrm{tr}}\right] \epsilon_{k} d$ and $\epsilon_{k}$ is the extinction coefficient of ${ }^{1} K_{\mathrm{tr}}$ at the monitoring wavelength. Thus, the decay kinetics of the composite absorbance

$$
A_{\mathrm{obs}}(t)=A_{k}(t)+A_{t}(t)
$$

is quite complex in dry $3 \mathrm{MP}$, and since the absorption spectra of the two components overlap (cf. Figure 3 ) and the secondorder rate constants $\theta_{2}{ }^{k}$ and $\theta_{2}{ }^{t}$ are quite similar in value, it is not possible to determine $k_{1}, \theta_{2}{ }^{k}$ and $\theta_{2}{ }^{t}$ in dry 3MP, i.e., to distinguish kinetically between the two transients. In watercontaining 3MP the kinetic analysis is less complicated because after $\sim 0.5 \mu$ s (cf. Figure 2C) the contribution of the protonquenched trans-keto tautomer to the absorbance becomes negligible and one can determine with good accuracy the triplet rate constants $k_{1}$ and $\theta_{2}{ }^{t}$ from traces with low time resolution (cf. Figure 2B). Assuming that the triplet decay is the same in dry 3MP, these values were used as fixed parameters for a fit of the composite decay law resulting from eqs $1-3$ to the $A_{\text {obs }}$ 
TABLE 1: Relative Quantum Yields, $\Phi_{\text {rel, }}$ of Trans-Keto Formation at Different Temperatures

\begin{tabular}{clll}
\hline$T$ & $\Phi_{\text {rel }}$ & $T$ & $\Phi_{\text {rel }}$ \\
\hline 280 & 1 & 220 & 0.26 \\
270 & 0.89 & 210 & 0.16 \\
260 & 0.76 & 200 & 0.10 \\
250 & 0.63 & 190 & 0.06 \\
240 & 0.50 & 180 & 0.03 \\
230 & 0.38 & 170 & 0.01
\end{tabular}

decay in dry 3MP (Figure 2A). It is not the purpose of this fit to provide an accurate value of $\theta_{2}{ }^{k}$ but rather to show the approximate time dependence of the quite substantial ${ }^{1} K_{\mathrm{tr}}$ absorbance contribution (dotted line in Figure 2A) to the total absorbance $A_{\mathrm{obs}}(t)$. To determine $\theta_{2}{ }^{k}$ accurately, it would be necessary to investigate dry air- or oxygen-equilibrated solutions.

The reason why we did not recognize earlier ${ }^{3.4}$ the presence of the oxygen-insensitive transient ${ }^{1} K_{t r}$ is embarrassingly trivial: To find out whether the transient species was quenched by oxygen, we equilibrated a dried 3MP/HBO solution with air simply by opening the sealed cell. With this procedure not only the triplet lifetime was shortened by the dissolved oxygen, but also the trans-keto lifetime by the introduction of moisture, resulting in a decay curve similar to Figure 2D which we misinterpreted as oxygen-quenched triplet decay. If one performs the quenching experiment properly with dried oxygen or air, only about one-quarter of the transient absorption is quenched.

The lifetime of ${ }^{1} \mathrm{~K}_{\mathrm{tr}}$ in water-containing 3MP (cf. Figure $2 \mathrm{C}$ ) is $\tau\left({ }^{1} \mathrm{~K}_{\mathrm{tr}}\right)=0.25 \mu \mathrm{s}$. This value is very close to $\tau_{\mathrm{a}}=0.26 \mu \mathrm{s}$ found, as mentioned above, by Itoh and Fujiwara. ${ }^{2}$ We therefore presume that the 3MP solution investigated in ref 2 contained traces of water. This would imply that the absorption and, in two-step-laser-excitation (TSLE) experiments, the emission of the proton-quenched trans-keto tautomer had been observed ${ }^{2}$ and not that of the cis-keto tautomer.

The absorption spectra of the two transients are shown in Figure 3. They were determined with a water-containing solution from traces like the one in Figure 2C. The spectra agree with those published by Itoh and Fujiwara (Figure 2 of ref 2) which supports our assumption that in this work, too, the trans-keto tautomer has been observed.

The yield of trans-keto formation decreases strongly at lower temperatures. From the difference of the absorbance amplitudes at zero time, $\mathrm{A}^{\circ}{ }_{\text {obs }}-A^{\circ}{ }_{t}=A^{\circ}{ }_{k}$, obtained from curves like those shown in Figure $2 \mathrm{C}$, the temperature dependence of the relative trans-keto yield, $\Phi_{\text {rel }}=A^{\circ}{ }_{k}(T) / A^{\circ}{ }_{k}(280 \mathrm{~K})$, has been determined and listed in Table 1 . Evidently, $\Phi_{\text {rel }}$ becomes virtually zero below $\sim 160 \mathrm{~K}$. Since, in addition, the triplet yield increases strongly at lower temperatures, ${ }^{3}$ the contribution of the trans- keto tautomer to the total absorbance $A_{\text {obs }}(T)$ becomes negligible below $\sim 200 \mathrm{~K}$. Thus, the $293 \mathrm{~K}$ absorption spectrum published by us in ref 3 (Figure 1) consists mainly of ${ }^{1} \mathrm{~K}_{\mathrm{tr}}$, the $201 \mathrm{~K}$ spectrum almost entirely of ${ }^{3} \mathrm{HBO}^{*}$. If one determines the transient spectrum with low time resolution $(10 \mu$ s in ref 4$)$, most of the trans-keto tautomer has decayed, as the calculated ${ }^{1} \mathrm{~K}_{\mathrm{tr}}$ and ${ }^{3} \mathrm{HBO} *$ absorbance curves in Figure $2 \mathrm{~A}$ show, and one observes also at higher temperatures mainly the triplet spectrum (cf. Figure 2 in ref 4 ).

\section{Conclusion}

HBO forms after ESIPT, like HBC and HBT, with high quantum yield the trans-keto tautomer. In inert solutions this transient decays in a second-order reaction. Therefore, it has been confused ${ }^{3}$ with the absorbance of the HBO triplet state which, in part, decays by second-order triplet-triplet annihilation. Traces of water shorten only the trans-keto lifetime and make the two transients easily distinguishable in nanosecond flash experiments. The contribution of the trans-keto tautomer to transient absorption signals becomes negligible below $\sim 200$ $\mathrm{K}$ so that the transient absorbance studies of proton transfer processes in the triplet state $e^{4.10 .11}$ at lower temperatures are not seriously affected. At ambient temperatures, however, the presence of the trans-keto tautomer has to be taken into account in kinetic studies. We presume that in the TSLE experiments of Itoh and Fujiwara ${ }^{2}$ the emission of the (proton-quenched) trans-keto tautomer has been observed, and not that of the cisketo tautomer. The latter is presumably very short-lived and can therefore not accumulate in detectable concentrations in flashed solutions of $\mathrm{HBO}$.

\section{References and Notes}

(1) For a recent review see: Ormson, S. M.; Brown, R. G. Prog. React. Kinet. 1994, 19, 45.

(2) Itoh, M.; Fujiwara, Y. J. Am. Chem. Soc. 1985, 107, 1561

(3) Mordzinski, A.; Grellmann, K. H. J. Phys. Chem. 1986, 90, 5503.

(4) Grellmann, K. H.; Mordzinski, A.; Heinrich, A. Chem. Phys. 1989. $136,201$.

(5) Rodriguez Prieto, M. F.; Nickel, B.; Grellmann, K. H.; Mordzinski, A. Chem. Phys. Lett. 1988, 146, 387.

(6) Brewer, W. E.; Martinez, M. L.; Chou, P. J. Phys. Chem. 1990, 94, 1915

(7) Al-Soufi, W.; Grellmann, K. H.; Nickel, B. Chem. Phys, Lett. 1990. 174,609 .

(8) Chou, P. T.; Martinez, M. L.; Studer, S. L. Chem. Phys. Lett. 1992. $195,586$.

(9) Stephan, J. S.; Rios Rodriguez, C.; Grellmann, K. H.; Zachariasse, K. A. Chem. Phys. 1994, 186, 435 .

(10) Al-Soufi, W.; Grellmann, K. H.; Nickel, B. J. Phys. Chem. 1991. $95,10503$.

(11) Eisenberger, H.; Nickel, B.; Ruth, A. A.; Al-Soufi, W.; Grellmann. K. H.; Novo, M. J. Phys. Chem. 1991, 95, 10509.

JP950340K 